\title{
Tazarotene gel with narrow-band UVB phototherapy: a synergistic combination in psoriasis ${ }^{*}$
}

\author{
Surabhi Dayal ${ }^{1}$ \\ Priyadarshini Sahu ${ }^{1}$
}

\author{
Rajiv Kaura ${ }^{2}$ \\ Vijay Kumar Jain ${ }^{1}$
}

DOI: http:/ / dx.doi.org/10.1590/abd1806-4841.20186723

Abstract: BACKGROUND: Narrow-band UVB (NB-UVB) has been shown to be one of the most effective treatment modalities for psoriasis. Tazarotene, a known effective anti-psoriatic modality, when combined with NB-UVB may enhance the therapeutic success.

OвјестіV: To study clinical efficacy and safety of combination of NB-UVB with topical tazarotene $0.05 \%$ gel in psoriasis.

MethoD: Thirty patients with plaque psoriasis having symmetrical lesions were enrolled for 12 weeks. All patients were instructed to apply tazarotene gel on target plaque on left side of body once daily. In addition, the whole body was irradiated with NB-UVB twice weekly. Efficacy was assessed by target plaque scoring and number of treatment sessions for clearance.

RESUlt: Our study resulted in 3 key findings: Firstly, therapeutic efficacy of NB-UVB was enhanced by addition of tazarotene. This enhanced efficacy was more apparent in decreasing scaling and thickness as compared to decrease in erythema. Secondly, combination therapy showed faster clearance of target plaques, with reduction in mean number of treatment sessions. Thirdly, mean cumulative NB-UVB dose needed to achieve clearance of target plaques was significantly reduced with combination therapy.

STUDY LIMITATIONS: The study was not randomized or controlled, but an open-label trial. The study period was relatively short, i.e., 12 weeks, without any follow-up period.

Conclusion: Tazarotene gel significantly enhances the therapeutic efficacy of NB-UVB irradiation with faster clearance and without serious side effects.

Keywords: Phototherapy; Psoriasis; Retinoids

\section{INTRODUCTION}

Psoriasis is a common, genetically determined chronic inflammatory dermatological disorder which affects skin, nails, and joints and has various systemic associations. According to the literature, the prevalence varies from $0.91 \%-8.5 \%$ in various populations ${ }^{1}$ and causes considerable morbidity and mortality. Psoriasis usually has a chronic course with periods of remission and exacerbation. However, no drug that completely cures psoriasis has been discovered to date. Various topical and systemic agents can provide symptomatic relief to the patient and prolong remission. Topical agents include topical steroids, dithranol (anthralin), tar preparations, topical retinoids, salicylic acid, and vitamin D derivatives. ${ }^{1}$

Phototherapy such as narrow band UVB (NB-UVB) is the recommended first-line therapy for most patients with moderate to severe psoriasis. ${ }^{1}$ However, in an attempt to enhance the efficacy and minimize the cumulative radiation dose, NB-UVB has been combined with other treatment modalities including dithranol, calcipotriol, tacalcitol, and oral retinoids. ${ }^{2-6}$ Calcipotriol when used in combination with phototherapy has been associated with photosensitivity. ${ }^{4}$ Although combination therapy with oral retinoids is known to enhance the efficacy of phototherapy, the prolonged use of such drugs is associated with a host of undesirable adverse effects including dry skin, headache, arthralgia, bone pain, hyperostosis, and teratogenicity. ${ }^{6}$

With the approval of tazarotene, the first receptor-selective topical retinoid indicated for treatment of psoriasis, the opportunity arose to investigate whether tazarotene improves the efficacy and tolerability of phototherapy. Combining NB-UVB with topical retinoids (tazarotene) would appear to be a better choice because tazarotene is not associated with the problems related to the use of psoralens, dithranol, calcipotriol, or oral retinoids. In a detailed search of

\footnotetext{
Received 18 November 2016.

Accepted 22 March 2017.

* Work conducted at the Pt B. D. Sharma, Post graduate Institute of Medical Sciences, Haryana, India.

Financial support: None.

Conflict of interest: None

Department of Dermatology, Venereology and Leprology, Pandit B.D. Sharma Post Graduate Institute of Medical Sciences, Rohtak, Haryana, India. Civil Hospital, Panchkula, Haryana, India.
}

MAILING ADDRESS:

Surabhi Dayal

E-mail: surabhidayal7@gmail.com 
the literature in English, to the best of our knowledge, there is only a single study comparing the combination of NB-UVB and tazarotene gel with NB-UVB alone in the treatment of psoriasis. ${ }^{7}$ This prompted us to undertake a comparative study of the clinical efficacy and safety of NB-UVB in combination with topical tazarotene gel 0.05\% versus NB-UVB alone in psoriasis, in terms of therapeutic efficacy, time to clear, and adverse effects.

\section{METHODS}

\section{Study Design:}

This was a 12-week, open-label, prospective, right-left intra-individual clinical study performed in the Department of Dermatology.

\section{Patient selection:}

Thirty patients 16 years and older with chronic stable plaque psoriasis not exceeding $50 \%$ of body surface area, according to the rule of nine, were included in this study. The disease was considered to be stable when there was no enlargement of plaques and no new lesions within the last 6 months of the study period. All the patients gave verbal and written informed consent before enrolment.

Exclusion criteria were age under 16 years or over 60 years, pregnancy, breastfeeding, renal or hepatic disease, previous carcinoma of skin or actinic keratosis, uncontrolled systemic diseases including HIV infection, or any immunosuppressive therapy. Patients with a history of oral retinoid use in the previous 8 weeks, active systemic therapy/PUVA therapy in the previous 4 weeks, topical therapy/UVB phototherapy in the previous 2 weeks, vitamin A supplements (>5000 IU) in the previous week, photosensitivity or polymorphic light eruption or history of intolerance or previous failure to phototherapy were also excluded from the study.

A detailed history was taken regarding age at onset, family history, duration of disease, and history of precipitating or initiating factors. Detailed dermatological examination including target plaque scoring was performed, and presence of any other associated dermatological condition was noted.

\section{Treatment schedule:}

In all the patients, two target plaques of psoriasis of approximately the same size were selected from similar sites on the right and left sides of the body. The target plaques on the right side were termed as Group A and were treated with topical tazarotene plus NB-UVB. Patients were instructed to apply a thin film of tazarotene $0.05 \%$ gel to the target plaque on the right side every evening. Similarly, the target plaques on the left side were termed as Group B and were treated with NB-UVB alone. No topical treatment was given on the target plaque on the left side of body. In addition, the whole body was irradiated with NB-UVB phototherapy twice a week on non-consecutive days for 3 months.

Whole-body NB-UVB (V care UV therapy unit, Bangalore, India) of wavelengths from 310 to $315 \mathrm{~nm}$, containing 16 fluorescent tubes (TL-01) with 100 watts and $6 \mathrm{ft}$. was used. Standard initial dose was $280 \mathrm{~mJ} / \mathrm{cm}^{2} .{ }^{8}$ The irradiation dose was increased by $20 \%$ over the previous dose on each subsequent visit. The UVB dose was increased until improvement was observed in the psoriasis plaque or the minimal erythema dose was reached. In case of symptomatic erythema with or without edema/blistering, further treatment was withheld until symptoms subsided. After resolution of symptoms, dose administration was reduced to $50 \%$ of the last dose and subsequent increments were by $10 \%$. The side which achieved clearance first was occluded and NB-UVB was continued on the other side till clearance of target plaque or 12 weeks of study completion, whichever was earlier. In a study by Hecker et al. ${ }^{9}$, tazarotene gel was found to remain chemically stable when applied before phototherapy (i.e. UVB or PUVA). However, in our study, it was applied after phototherapy to ensure that the response due to therapy was not confounded by any possible effect that tazarotene gel might have on ultraviolet light penetration. ${ }^{10}$

During each treatment, eyes were protected by UV blocking goggles, and genitalia and other uninvolved areas were shielded. All patients were asked to avoid direct UV exposure and use sunscreen during daytime.

\section{Clinical assessment:}

- Primary efficacy criterion was the Target Plaque Severity Score $^{11}$

The assessment was done on the basis of morphological scoring of target plaques by evaluation of three parameters, i.e. erythema, scaling, and thickness, each of which was graded on a severity scale of 0 to 4 , where
0 - Nil
1- Mild
2- Moderate
3- Severe
4- Very severe

The sum of the scores for each parameter gave the target plaque score. The target plaque score had a potential range of 0-12. This scoring was used since it is part of the PASI (Psoriatic Area Severity Index) and did not include the localization and extent, which are not considered useful in an intra-individual comparison with a limited body surface area involvement. ${ }^{12}$ All the patients were examined by the same dermatologist at each visit up to 3 months. Target plaque scoring was done at baseline and then at 4, 8, and 12 weeks of therapy separately for both target plaques. In addition, individual scoring of erythema, scaling, and thickness was also done for each target plaque.

Secondary efficacy criteria were:

a) To determine the percentage of patients who achieve "treatment success", defined as $\geq 50 \%$ reduction in target plaque score from baseline with therapy. ${ }^{9}$

b) To determine the mean cumulative dose of NB-UVB, number of treatment sessions, and total treatment period required for target plaque clearance (i.e. target plaque score $=0$ ).

c) To evaluate the overall treatment response, graded as: $:^{10}$

$0=$ Completely cleared

$1=$ Almost cleared ( 90\% improvement)

$2=$ Marked response $(\sim 75 \%$ improvement $)$

$3=$ Moderate response ( $50 \%$ improvement)

$4=$ Slight response $(\sim 25 \%$ improvement $)$

$5=$ Condition unchanged 
$6=$ Condition worsened

d) To document the adverse effects at each visit, such as lesional irritation, burning sensation, itching etc.

\section{Statistical analysis:}

All statistical analyses were carried out with Statistical Package for Social Sciences (SPSS) for Microsoft Windows, $20^{\text {th }}$ version. Descriptive statistics were analyzed on the parameters of range, mean \pm standard deviation, frequencies (number of cases), ratio, or percentages, as appropriate. The changes in the parameters such as target plaque score, target plaque erythema, thickness, and scaling between the two groups and within the groups were analyzed using Mann-Whitney and Wilcoxon signed-rank test, respectively. Similarly, the difference in the number of treatment sessions and mean cumulative dose between the target plaques was compared using Mann-Whitney test. The side effects between the two groups were compared using chi-square test. P-value $<0.05$ was considered statistically significant.

\section{RESULTS}

\section{Baseline patient characteristics:}

Table 1 shows the demographics of 30 patients. The target plaque score of the two target plaques selected in each patient did not differ significantly between the two sides at baseline $(p>0.05)$. Similarly, there was no statistical difference in the mean baseline target plaque erythema, scaling, or thickness ( $p>0.05)$ and hence the two target plaques were comparable before initiating therapy.

\section{Assessment of Clinical Efficacy:}

a) Target Plaque Scaling - At baseline, target plaque scaling in Group A was $2.47 \pm 0.68$ and in Group B was $2.46 \pm 0.69$. Thus, the difference in target plaque scaling was not statistically significant ( $p>0.05$ ) before starting therapy. The difference between the mean target plaque scaling was found to be highly significant in Group A as compared to Group B at 4, 8, and 12 weeks of therapy (Figure 1A). At the end of therapy, the mean value for plaque scaling was reduced by $98.7 \%$ in Group A as compared to $78.54 \%$ in Group B.

b) Target Plaque Thickness - Before starting therapy, target plaque thickness in Group A was $3 \pm 0.64$ and in Group B was 3.03 \pm 0.6 . Thus, the difference was not statistically significant $(\mathrm{p}>0.05)$ at baseline. During the course of treatment, the difference in target plaque thickness between the two groups was found to be statistically significant in Group A as compared to Group B at 4, 8, and 12 weeks (Figure 1B). At the end of 12 weeks, the mean value for

TABLE 1: Demographic data of patients included in the study

Demographic Data

Patients

Age (years) Mean + SD

$36.40+12.552$

Sex ratio (M: F)

$7: 3$ [Male $=21$, Female $=9]$

Duration of disease (years) Mean $5.8+3.112$

$+\mathrm{SD}$

Body surface area affected (Percent- $\quad 25.97+5.379$ age; \%) Mean + SD plaque thickness was reduced by $99.9 \%$ in Group A as compared to $79 \%$ in Group B.

c) Target Plaque Erythema - At baseline, target plaque erythema in Group A was $2.96 \pm 0.61$ and in Group B was $2.86 \pm 0.68$, which was not statistically significant $(\mathrm{p}>0.05)$. During therapy, there was slightly more erythema over target plaques treated with NB-UVB plus tazarotene compared to target plaques treated with NB-UVB alone. Thus, the difference was not statistically significant during the whole course of treatment. However, erythema became negligible at the end of treatment in both target plaques (as shown in Figure 2A). At the end of therapy, mean reduction in target plaque erythema was nearly equal in both groups, i.e. $97.97 \%$ in Group A and $100 \%$ in Group B.

d) Target Plaque Score -Before starting therapy, target plaque score in Group A was $8.37 \pm 0.96$ and in Group B was $8.37 \pm$ 0.97 . Thus, the difference in target plaque scoring was not statistically significant $(\mathrm{p}>0.05)$, at baseline. During the course of therapy, the difference in target plaque score between the two target plaques became statistically significant after 4 weeks of therapy $(p<0.05)$. At the end of 12 weeks, the mean target plaque score was reduced by $99.64 \%$ in Group A as compared to $84.92 \%$ in Group B (as shown in Figure 2B).

e) Treatment Success- At 4 weeks of therapy, $83.33 \%$ of target plaques in Group A and only 3.33\% of target plaques in Group B achieved treatment success. At 8 weeks, $100 \%$ of target plaques in Group A and $86.67 \%$ of target plaques in Group B achieved treatment success. At the end of therapy, treatment success was achieved in $100 \%$ of target plaques in both groups. The number of days to achieve treatment success in target plaques receiving only NB-UVB therapy $(65.30 \pm 8.469)$ was more than in target plaques treated with NB-UVB plus tazarotene (31.96 \pm 5.486$)$. The difference was statistically significant $(\mathrm{p}<0.05)$.

f) Target Plaque Clearance- The numbers of treatment sessions required for clearance of target plaques in Group A and B were $16.40 \pm 2.799$ and $23.33 \pm 1.212$, respectively. Thus, the number of treatment sessions required for clearance of target plaques in Group A was significantly lower than in Group B ( $p<0.05)$. The mean cumulative dose for clearance in Group B was $4010.267 \pm 784.39 \mathrm{~mJ} /$ $\mathrm{cm}^{2}$, while in Group A it was $1839.533 \pm 259.326 \mathrm{~mJ} / \mathrm{cm}^{2}$. Thus, the mean cumulative dose for clearance was significantly lower in target plaques treated with NB-UVB plus tazarotene as compared with the target plaques treated with NB-UVB alone $(p<0.05)$.

g) Overall treatment response- According to assessment of overall response at the end of treatment, $96.67 \%$ of target plaques in Group A achieved complete clearance, i.e. grade 0, while only $6.67 \%$ of target plaques in Group B achieved complete clearance. Another 3.37\% of target plaques in Group A achieved marked response, i.e. $\sim 75 \%$ improvement, while $93.33 \%$ of target plaques in Group B achieved marked response. None of the target plaques in either group had become worse. Figures 3 and 4 are photographs of two patients showing sequential improvement in target plaques.

\section{Safety Evaluation:}

All patients developed grade I erythema (i.e. asymptomatic faint and barely perceptible erythema) ${ }^{13}$ Grade II erythema (i.e. 
moderate and well-defined asymptomatic erythema) was observed in an equal number of target plaques, i.e. 4 in both the groups after 8 weeks of treatment. ${ }^{13}$ In all these patients, treatment was stopped until the erythema subsided and NB-UVB was started with the previous tolerable dose, with subsequent $10 \%$ increments according to protocol. Only one patient developed perilesional tenderness on both sides. Tazarotene was well tolerated except for lesional irritation observed in only $2(6.67 \%)$, burning sensation in $1(3.33 \%)$, and pruritus in $2(6.67 \%)$ target plaques treated with combination therapy. These were mild in severity and were typical effects associated with retinoid therapy. Thus, on comparing the adverse effects in the two groups, Group A was associated with more side effects in contrast to Group B, but the difference was not statistically significant $(p=0.352)$.

\section{DISCUSSION}

Phototherapy, especially NB-UVB, has proven to be one of the most effective treatment modalities for patients with psoriasis. Although NB-UVB is often effective in improving extensive moderate-to-severe psoriasis, even maximally aggressive treatment may fail to completely clear psoriatic lesions. Thus, photo-combination therapies like NB-UVB with dithranol, calcipotriol, tacalcitol, and oral retinoids, which are capable both of reducing cumulative UV
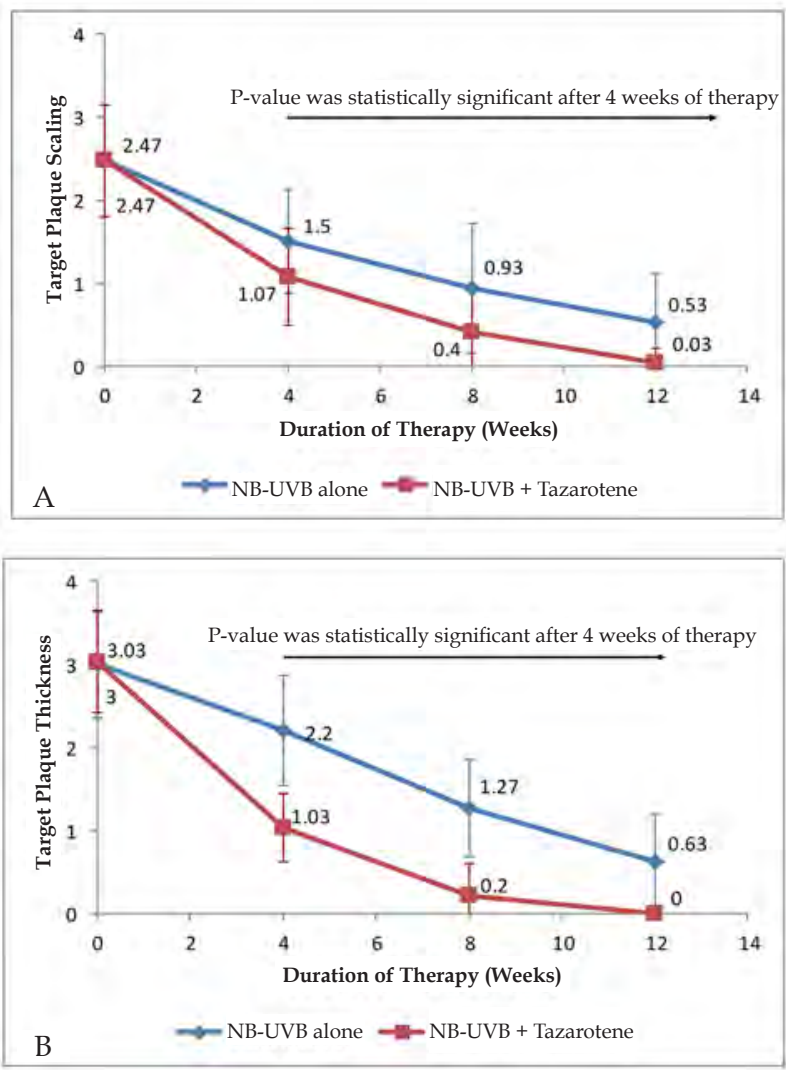

FigURE 1: A: Graph showing variation in scaling over target plaques from baseline when treated with tazarotene $0.05 \%$ gel plus NB-UVB phototherapy versus NB-UVB phototherapy alone.

B: Reduction in target plaque thickness from baseline after treatment with tazarotene $0.05 \%$ gel plus NB-UVB phototherapy versus NBUVB phototherapy alone doses and of accelerating clearance of skin lesions, are important and of high interest currently. ${ }^{2-6}$ However, because the majority of these therapeutic modalities used in combination with NB-UVB have major limitations, a new treatment modality is still needed that is suitable for combination with NB-UVB by enhancing the long and short-term antipsoriatic effectiveness of NB-UVB and improving its side-effect profile.

Tazarotene is the first member of a novel class of topical retinoids. Studies suggest that tazarotene ameliorates the three major pathogenic factors in psoriasis, i.e. keratinocyte hyperproliferation, abnormal keratinocyte differentiation, and infiltration of inflammatory cells. ${ }^{7}$ Moreover in patients with plaque psoriasis, tazarotene had significantly better maintenance of therapeutic effects after cessation of therapy in comparison to potent topical corticosteroids. ${ }^{14}$ A recent study by Luo et al. ${ }^{15}$ suggested that a single treatment with tazarotene can inhibit keratinocyte proliferation and increase TIG3 (tazarotene-induced gene) mRNA expression, but NB-UVB irradiation only inhibits cell proliferation at a higher dose. However, when NB-UVB is combined with tazarotene gel it has synergistic effects on inhibiting keratinocyte proliferation and elevating in vitro TIG3 expression. Thus, this study has shown that when NB-UVB is used in combination with tazarotene, it exhibits a stronger effect as compared to their use as monotherapies. Therefore, we undertook a clin-
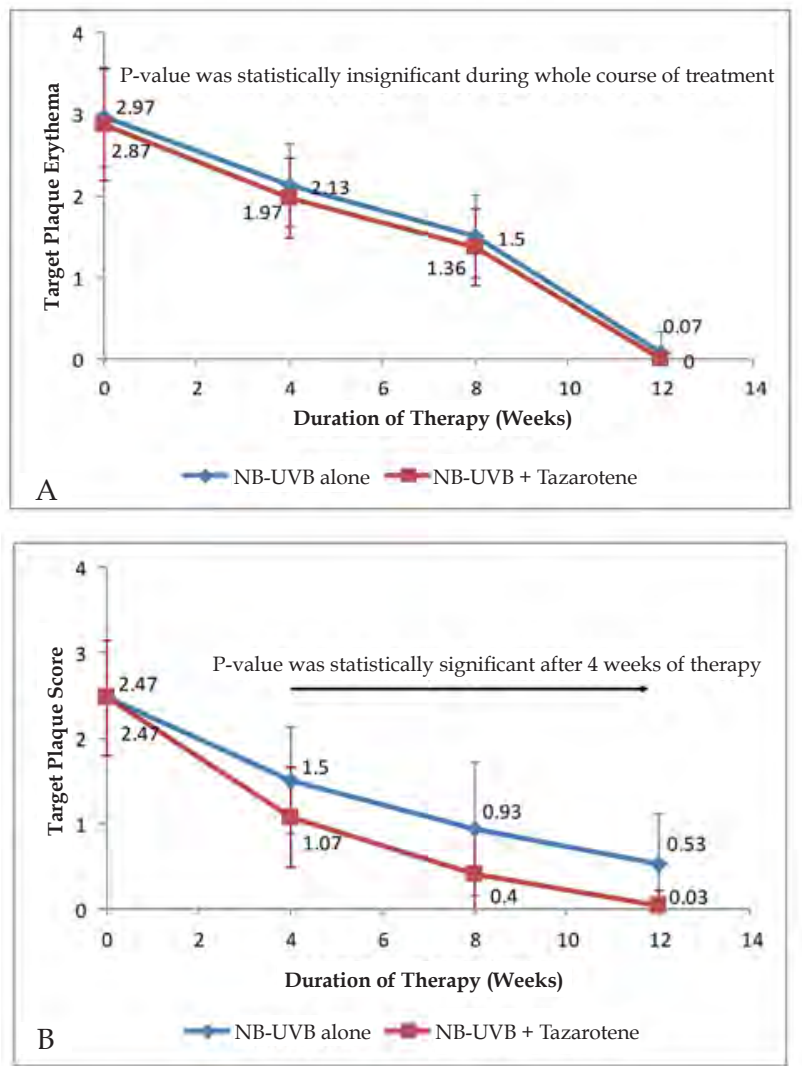

FIGURE 2: A - Graph showing variation in erythema over target plaques from baseline when treated with tazarotene $0.05 \%$ gel plus NBUVB phototherapy versus NB-UVB phototherapy alone. B - Reduction in target plaque score from baseline when treated with tazarotene $0.05 \%$ gel plus NB-UVB phototherapy versus NBUVB phototherapy alone 
ical trial to study the therapeutic efficacy and safety of combining tazarotene with NB-UVB in plaque type psoriasis.

In our study, when the target plaques were clinically evaluated after therapy, we observed a statistically significant decrease in target plaque score on the side treated with NB-UVB plus tazarotene as compared to the side treated with NB-UVB alone. This corroborated the study by Behrens et al. ${ }^{7}$, in which efficacy was assessed by means of modified PASI score, showing that after 4 weeks there was a statistically significant reduction in median PASI score with combination therapy as compared to NB-UVB alone. Thus, clinical trials combining tazarotene with NB-UVB and enrolling more patients with longer follow-up are lacking in the literature.

We further compared the efficacy of the two regimens by studying separately the reduction in target plaque thickness, scaling, and erythema. There was a significantly greater and faster reduction in plaque thickness and scaling from baseline in target plaques treated with NB-UVB plus tazarotene as compared to target plaques treated with NB-UVB alone after 8 weeks of therapy. Meanwhile, erythema was slightly more frequent in target plaques treated with NB-UVB plus tazarotene, compared to those treated with NB-UVB alone. This may have been due to tazarotene-induced skin irritation, which was reported in $23 \%$ of patients treated with tazarotene alone in a study by Weinstein et al. ${ }^{16}$ However, in our study, the difference between the two groups as to lesional erythema was not statistically significant, and became negligible by the end of therapy in both groups. This may have been due to the fact that UV irradiation reduces this adverse effect by enhancing the skin barri- er function, making it more resistant to irritants when compared to non-irradiated skin, as demonstrated by Lehmann et al. ${ }^{17}$

According to our study, the number of days required for initial treatment success in target plaques treated with combination therapy was significantly lower than for target plaques treated with NB-UVB alone. Likewise, the number of treatment sessions and mean cumulative dose of phototherapy required for clearance of psoriatic plaques was significantly lower with combination therapy as compared to NB-UVB alone. This agrees with the study by Behrens et al. ${ }^{7}$, who found that administration of topical tazarotene enhanced the therapeutic efficacy of NB-UVB irradiation, with reduction of mean number of treatment sessions and lower cumulative UVB dosage. The total duration of disease varied from 2 to 12 years in our study. However, no effect of duration of disease was seen as far as the response to treatment was concerned. The total duration seen in our study was in agreement with the study by Koo et $a l .{ }^{10}$. But none of the studies had evaluated the effect of duration of disease on treatment response.

When compared to the earlier study by Behrens et al., our study included considerably more patients (i.e. 30) and gave irradiation only two days a week on non-consecutive days, in contrast to Behrens et al., who included only 10 patients for 4 weeks and gave NB-UVB irradiation five times a week. Patient follow-up was also longer in our study, i.e. 12 weeks as compared to Behrens et al. ${ }^{7}$ Behrens et al. ${ }^{7}$ also used the modified PASI score to assess therapeutic efficacy and did not comment on the effect of therapy on individual parameters like plaque erythema, scaling, and thickness.

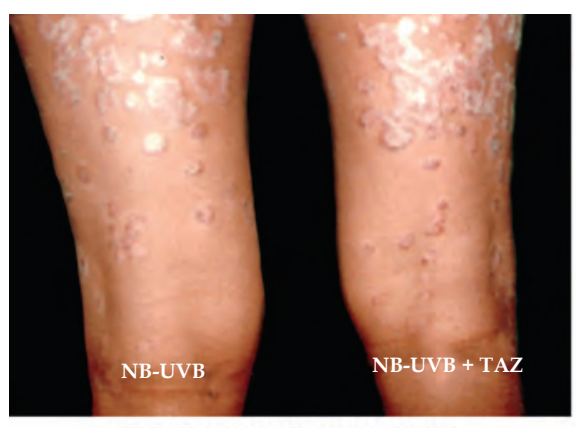

AT THE START OF TREATMENT

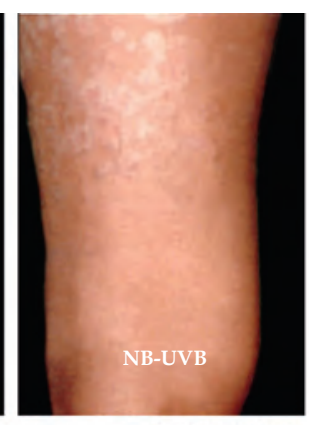

AT 6 WEEKS OF TREATMENT
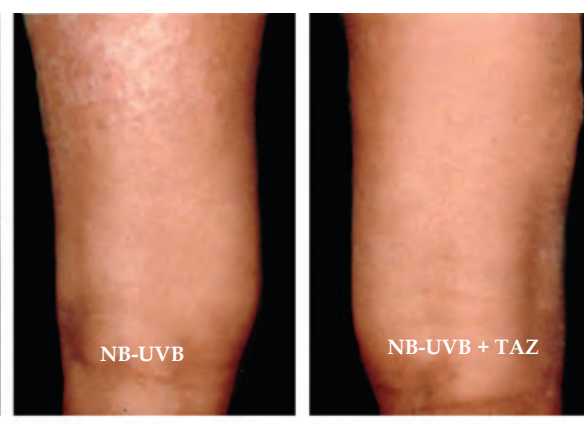

AT 12 WEEKS OF TREATMENT

FIGURE 3: Photographs of target plaques located on the posterior aspect of thighs at the beginning and end of therapy

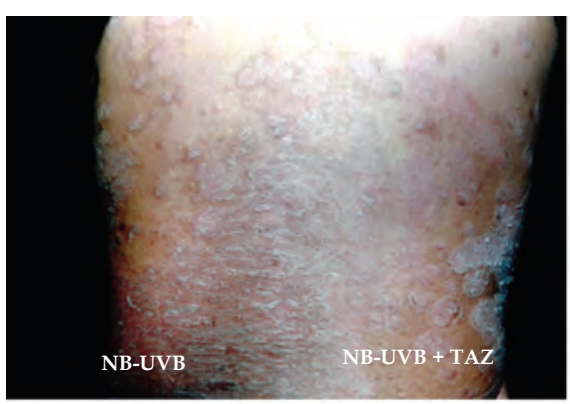

AT THE START OF TREATMENT

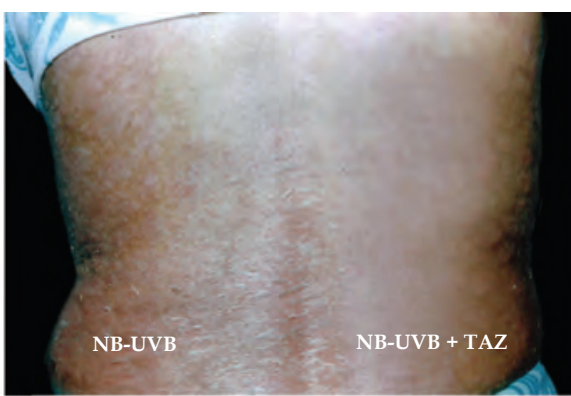

AT 6 WEEKS OF TREATMENT

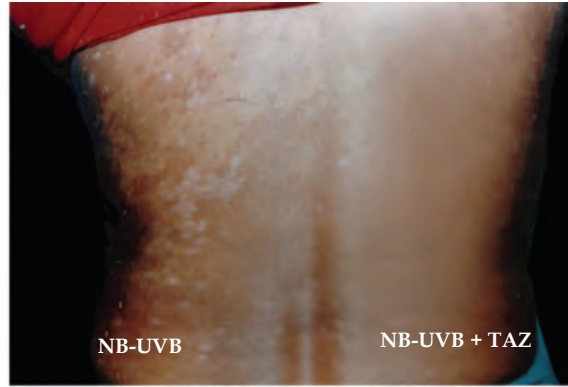

AT 12 WEEKS OF TREATMENT

FIGURE 4: Photographs of target plaques located on the back at the beginning and end of therapy 
Meanwhile, we chose target plaque scores to evaluate treatment outcome in order to avoid the influence of factors like body surface area involvement on therapeutic efficacy and to monitor the effect of this combination therapy on individual parameters like plaque erythema, scaling, and thickness.

The adverse effects experienced by all patients on both sides were mild and did not require permanent discontinuation of the treatment. Moreover, there were no differences in adverse effects between the two sides. Thus, the safety profile did not vary when tazarotene was used with NB-UVB. The side effect profile observed in our study is in agreement with Behrens et al. ${ }^{7}$ who also showed that both modalities were well tolerated and that adverse effects were limited to mild irritation and transient burning.

\section{REFERENCES}

1. Burden DA, Kirby B. Psoriasis and related disorders. In: Griffiths CEM, Barker J, Bleiker T, Chalmers R, Creamer D, editors. Rook's Textbook of Dermatology. 9th ed. Oxford: Wiley Blackwell;2016. p.35.1-35.29.

2. Carrozza P, Häusermann P, Nestle F0, Burg G, Böni R. Clinical efficacy of narrowband UVB $(311 \mathrm{~nm})$ combined with dithranol in psoriasis. An open pilot study. Dermatology. 2000:200:35-9.

3. $\operatorname{Rim} \mathrm{JH}$, Choe YB, Youn Jl. Positive effect of using calcipotriol ointment with narrow-band ultraviolet B phototherapy in psoriatic patients. Photodermatol Photoimmunol Photomed. 2002;18:131-4.

4. McKenna KE, Stern RS. Photosensitivity associated with combined UV-B and calcipotriene therapy. Arch Dermatol. 1995;131:1305-7.

5. Messer G, Degitz K, Plewig G, Röcken M. Pretreatment of psoriasis with vitamin D3 derivative tacalcitol increases the responsiveness to 311-nm ultraviolet $B$ : Results of a controlled, right/left study. Br J Dermatol. 2001;144:628-9.

6. Olsen EA, Weed WW, Meyer CJ, Cobo LM. A double-blind, placebo-controlled tria of acitretin for the treatment of psoriasis. J Am Acad Dermatol. 1989;21:681-6.

7. Behrens S, Grundmann-Kollmann M, Schiener R, Peter RU, Kerscher M.. Combination phototherapy of psoriasis with narrow-band UVB irradiation and topical tazarotene gel. J Am Acad Dermatol. 2000;42:493-5

8. Rai R, Srinivas CR. Phototherapy: an Indian perceptive. Indian J Dermatol 2007; 52:169-75.

9. Hecker D, Worsley J, Yueh G, Kuroda K, Lebwohl M. Interactions between tazarotene and ultraviolet light. J Am Acad Dermatol. 1999;41:927-30.
However, there were some limitations to our study. Firstly, the study was not randomized or controlled, but an open-label trial. Secondly, the study period was relatively short i.e., 12 weeks, without any longer follow-up. Thus, larger and longer-term prospective studies are needed to substantiate our study's findings.

\section{CONCLUSION}

We concluded from our study that combining NB-UVB with topical tazarotene gel $0.05 \%$ is a well-tolerated and effective antipsoriatic treatment strategy, as it significantly enhances the clinical efficacy, accelerates clearance, reduces the mean number of treatment sessions required, and lowers the mean cumulative clearance dose of NB-UVB irradiation. Tazarotene gel can also be a practical approach for patients with limited plaque type psoriasis who are treatment-resistant, as well as patients who cannot afford expensive biological agents.
10. Koo JY, Lowe NJ, Lew-Kaya DA, Vasilopoulos Al, Lue JC, Sefton J, et al. Tazarotene plus UVB phototherapy in the treatment of psoriasis. J Am Acad Dermatol. 2000;43:821-8.

11. Das S, Lloyd JJ, Walshaw D, Diffey BL, Farr PM. Response of psoriasis to sunbed treatment: comparison of conventional ultraviolet $A$ lamps with new higher ultraviolet B-emitting lamps. Br J Dermatol. 2002;147:966-72.

12. Van de Kerkhof PC, Werfel T, Haustein UF, Luger T, Czarnetzki BM, Niemann R, et al. Tacalcitol ointment in the treatment of psoriasis vulgaris: a multicentre, placebo-controlled, double-blind study on efficacy and safety. $\mathrm{Br} \mathrm{J}$ Dermatol. 1996;135:758-65

13. Dogra S, De D. Narrowband ultraviolet B in the treatment of psoriasis: The journey so far! Indian J Dermatol Venereol Leprol. 2010;76:652-61.

14. Esgleyes-Ribot T, Chandraratna RA, Lew-Kaya DA, Sefton J, Duvic M.. Response of psoriasis to a new topical retinoid, AGN 190168. J Am Acad Dermatol. 1994;30:581-90.

15. Luo S, Zheng Y, Peng Z, Jiang J, Gondokaryono S, Wang G, et al. Effects of Narrow-band ultraviolet $B$ and tazarotene therapy on keratinocyte proliferation and TIG3 expression. J Dermatol 2008; 35:651-7.

16. Weinstein GD, Krueger GG, Lowe NJ, Duvic M, Friedman DJ, Jegasothy BV,et al. Tazarotene gel, a new retinoid, for topical therapy of psoriasis: vehicle-controlled study of safety, efficacy, and duration of therapeutic effect. J Am Acad Dermatol. 1997; $37: 85-92$

17. Lehmann P, Hölzle E, Melnik B, Plewig G. Effects of ultraviolet A and B on the skin barrier: a functional, electron microscopic, and lipid biochemical study. Photodermatol Photoimmunol Photomed. 1991;8:129-34.

$\begin{array}{ll}\text { Surabhi Dayal } & \text { (iD) ORCID 0000-0001-9093-6731 } \\ \text { Rajiv Kaura } & \text { (D) ORCID 0000-0002-1273-447X }\end{array}$

$\begin{array}{ll}\text { Priyadarshini Sahu } & \text { (D) ORCID 0000-0002-7064-4181 } \\ \text { Vijay Kumar Jain } & \text { (D) ORCID 0000-0002-4486-9301 }\end{array}$

How to cite this article: Dayal S, Kaura R, Sahu P, Jain VK. Tazarotene gel with narrow-band UVB phototherapy: a synergistic combination in psoriasis. An Bras Dermatol. 2018;93(3):385-90. 